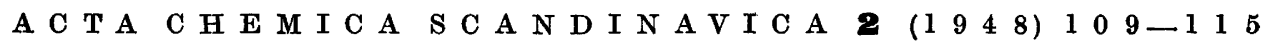

\title{
The Alkoxylation of Simple Furans and Related Reactions
}

\author{
NIELS CLAUSON-KAAS, FRANZ LIMBORG and \\ JØR G EN FAKSTORP
}

Chemical Laboratory of the University, Copenhagen, Denmark

B

the addition of alkoxy groups to the $\alpha$-carbons of furans, stable 2,5dialkoxy-2,5-dihydrofurans are obtained ${ }^{1}$. These addition products may be hydrolyzed under very mild conditions to the corresponding unsaturated 1,4-dicarbonyl compounds, which often are unstable and therefore difficult of accession. The procedure can be illustrated by the methoxylation of furan.

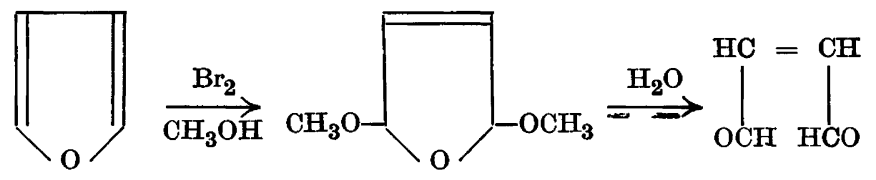

In this article we report some experiments concerning the general applicability of the alkoxylation method. In conclusion, other methods for the preparation of dihydrofurans by oxidation of furans are discussed.

\section{ALKOXYLATION}

Alkoxylation of furans is accomplished with bromine in alkohol at low temperature in the presence of potassium acetate, which neutralizes the hydrogen bromide formed by the reaction. Furan ${ }^{1}$, silvan ${ }^{2}$, furfuryl alcohol ${ }^{3}$, furfuryl acetate ${ }^{2}$, furfural ${ }^{3}$ and furfural diacetate ${ }^{4}$ have been methoxylated by this or a similar method. Furan has also been ethoxylated in the same way ${ }^{1}$.

We have tried to methoxylate some furans with electronegative substituents in the $\alpha$-positions, viz. furoic acid, ethyl furoate, ethyl furylacrylate, acetylfuran and 2,5-dibromofuran. None of the compounds yielded the corresponding dimethoxy derivatives. It therefore seems fairly safe to assume, 
that $\alpha$-substituted furans with substituents, which are known to decrease the reactivity of unsaturated systems towards the usual double bond reagents, as a rule do not yield addition products with bromine in methanol. The only furan derivative, with an electronegative $\alpha$-substituent, which has been methoxylated, is furfural $^{3}$. But in this case dimethoxydihydrofurfural dimethyl-acetal was formed, so that probably acetalization or semi-acetalization has taken place prior to methoxylation.

At present nothing is known about the applicability of the alkoxylation method on $\beta$-substituted furans.

It has previously been observed that a small amount of a higher boiling compound is obtained by the preparation of dimethoxydihydrofuran. This has now been identified as 2-methoxy-5-acetoxy-2,5-dihydrofuran. The structure was proved by analysis, methoxy, acetoxy and molecular weight determination, and by hydrolysis to malealdehyde. The formation of methoxyacetoxy-dihydrofuran together with dimethoxydihydrofuran may be explained as follows:
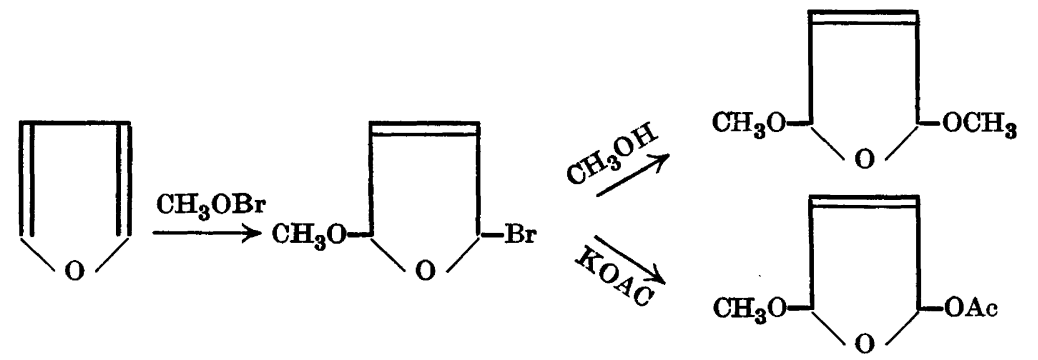

We have also investigated the alkoxylation of furan with higher alcohols, viz. isopropyl, $n$-butyl and isoamyl alcohol. The reaction mixture was not poured into a solution of calcium chloride, but sodium alcoholate was added to neutralize the acetic acid formed by the reaction, after which the mixture was distilled through a column. In this way the major part of the alcohols could be recovered. The acetals were formed in good yields. They are stable liquids, which can be distilled in vacuum. The structure was confirmed by hydrolysis to malealdehyde.

Dibutoxy- and diisoamyloxy-dihydrofuran have a pleasant odor, faintly resembling that of diisoamyl ether.

The molar refraction of the various dihydrofurans was determined, and a small negative exaltation of approximately the same size $(0.4-0.6)$ was found for all compounds. This is in agreement with the proposed similarity of structure. 


\section{RELATED REACTIONS}

Acetoxylation. Addition of acetoxy groups to furan has been performed with lead tetraacetate, ${ }^{1}$ and with bromine in acetic acid in the presence of potassium acetate ${ }^{5}$. No other furans have hitherto been acetoxylated.

It was to be expected that acetoxylation would be subject to the same limitation as alkoxylation. In agreement herewith, we were not able to add acetoxy groups to furoic acid or ethyl furoate by the above methods. But this is obviously not the only limitation of the methods, as silvan could not be acetoxylated with bromine in acetic acid either. The reaction product decomposed suddenly to a black mass when a distillation was attempted (at $1 \mathrm{~mm})$. We believe that decomposition is due to the formation of furfuryl bromide. This suggestion was further strengthened by the isolation from the reaction mixture of a small amount of furfuryl acetate.
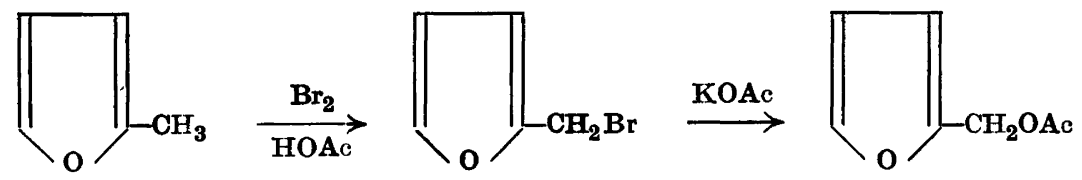

Buu-Hoì and Lecocq found, that silvan reacts with bromo succinimide in the same way ${ }^{6}$.

Marquis' method. Marquis found 7, that a nitro and an acetoxy group are added to furan and ethyl furoate by the action of nitric acid in acetic anhydride. The procedure has later been extended to other furans. The resulting nitroacetates may in some cases be hydrolyzed to the corresponding unsaturated 1,4-dicarbonyl compounds, but these can, as a rule, be obtained much easier from dialkoxydihydrofurans, because the nitroacetates often are labile and difficult to hydrolyze. They also very easily split off acetic acid with the formation of an $\boldsymbol{\alpha}$-nitrofuran. However, Marquis' method is of importance for the preparation of unsaturated 1.4-dicarbonyl compounds from furans because at present it is the only general way of obtaining 1,4-addition products of simple furans with an electronegative a-substituent. $\boldsymbol{E} . g$., oxidation of ethyl furoate and addition of hydroxylamine to the nitroacetate (I) yields a derivative (II) of the acid III (Marquis ${ }^{7}$ ). This compound cannot be obtained by any other method.

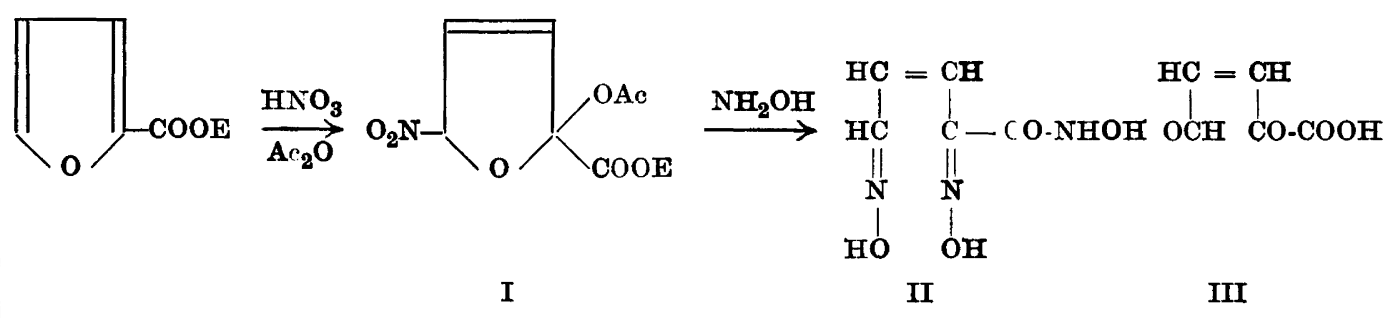


Oxidation with hydroxylamine. It has recently been observed ${ }^{8}$, that $p$ toluenesulphonyl 2-acetofuran-oxime reacts with alcohol to form the acetal IV and toluenesulphonic acidic ammonium. No details of the reaction mechanism are known, but the net result of the reaction is that hydroxylamine

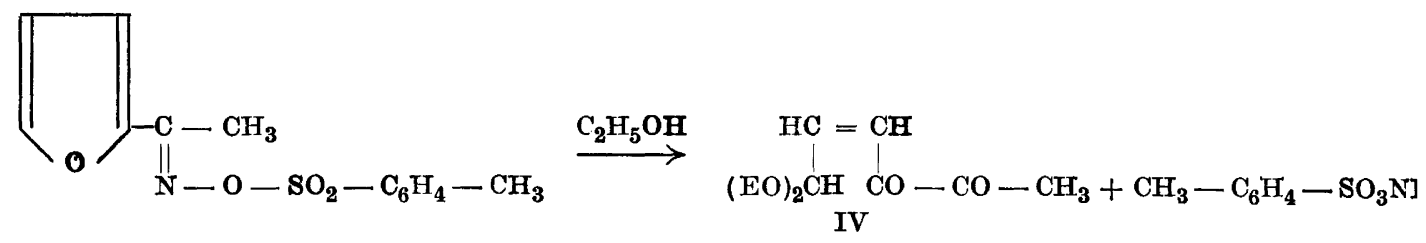

has oxidized acetofuran to the corresponding carbonyl compound. It is possible that the method is of general applicability for the oxidation of furans with a carbonyl group in the $\alpha$-position.

\section{EXPERIMENTAL}

2,5-Dimethoxy-2,5-dihydrofuran and $2-m e t h o x y-5-a c e t o x y-$ $2,5-d i h y d r o f u r a n$

$36 \mathrm{ml}$ of freshly distilled furan $(0.5 \mathrm{~mole})$ and $100 \mathrm{~g}$ of anhydrous potassium acetate ( $1 \mathrm{~mole}$ ) are dissolved in $600 \mathrm{ml}$ of methanol. The mixture is cooled in an ice-salt bath and a solution of $25 \mathrm{ml}$ of bromine in $500 \mathrm{ml}$ of methanol is added under efficient stirring. The temperature must be kept below $-7^{\circ}$. After all the bromine has been added ( 1 hour) stirring is continued for 5-10 minutes. Then the reaction mixture is poured into 1500 $\mathrm{ml}$ of a cooled, saturated, solution of calcium chloride. The acetal is extracted with 2000 $\mathrm{ml}$ of ether, and the etheral layer shaken with $250 \mathrm{ml}$ of a saturated solution of potassium carbonate and dried over $100+500 \mathrm{~g}$ of solid potassium carbonate. The ether is removed on the steam bath through a Vigreux column and the residue distilled in vacuum. Distillation begins at $54^{\circ} / 13 \mathrm{~mm}$ and the fraction boiling at $54-56^{\circ}$ is collected. It consists of dimethoxydihydrofuran, yield $42 \mathrm{~g}=65 \%$. Distillation is continued, whereby about $4 \mathrm{~g}$ of higher boiling material is obtained (b. p. 56-106 $6^{\circ}$. Redistillation of this fraction yields $3.2 \mathrm{~g}$ of a colourless liquid (b.p. ${ }_{13} 96-99^{\circ}$ ) which is analytically pure methoxyacetoxy-dihydrofuran. The compound turns yellow on prolonged standing but is otherwise stable.

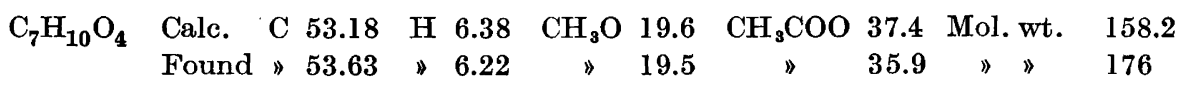

We have tried to carry out the synthesis of dimethoxydihydrofuran by adding sodium methoxide to the reaction mixture as described for the preparation of higher alkoxy derivatives, and also by substituting potassium acetate by pyridine. In both cases good yields of dimethoxydihydrofuran were obtained, but the method reported above was found to be more convenient. 
Hydrolysis of methoxy-acetoxy-dihydrofuran; identification of malealdehyde. $189 \mathrm{mg}$ of methoxy-acetoxy-dihydrofuran were boiled with $3 \mathrm{ml}$ of $0.01 \mathrm{~N}$ hydrochloric acid and $2 \mathrm{ml}$ of alcohol for one minute. After cooling, a solution of $0.3 \mathrm{ml}$ of phenylhydrazine in diluted acetic acid $(10 \mathrm{ml})$ was added and the bis-phenyl hydrazone of malealdehyde isolated in the usual manner ${ }^{1}$. Yield of crude product $209 \mathrm{mg}=66 \%$. Yield after recrystallization from acetone-benzene $151 \mathrm{mg}=48 \%$. M. p. 168-69 ${ }^{\circ}$ (Kofler stage, corr.), previously found ${ }^{9} 171^{\circ}$.

$$
\mathrm{C}_{16} \mathrm{H}_{16} \mathrm{~N}_{4} \text { (264) Calc. } \mathrm{N} 21.21 \quad \text { Found } \mathrm{N} 21.13
$$

Higher dialkoxy-dihydrofurans

$7.25 \mathrm{ml}$ of furan $(0.1 \mathrm{~mole})$ and $20 \mathrm{~g}$ of potassium acetate $(0.2 \mathrm{~mole})$ are dissolved, or suspended, in $130 \mathrm{ml}$ of the alcohol, (technical grade) and $5 \mathrm{ml}$ of bromine in $100 \mathrm{ml}$ of the alcohol are added at $-7^{\circ}$ during $20-30$ minutes, as described above. The reaction mixture is heated to room temperature and allowed to stand for 30 minutes. Then a solution of sodium alkoxide from $4.60 \mathrm{~g}$ of sodium $(0.2 \mathrm{~mole})$ is added at $0^{\circ}$ under stirring and efficient cooling. After 15 minutes $300 \mathrm{ml}$ of ether are added, and the precipitated potassium bromide and sodium acetate filtered off and washed with $50 \mathrm{ml}$ of ether. The ether is removed on the steam bath, and the residue distilled in vacuum through a Widmer column. Hereby the major part of the alcohol is recovered and may be employed in a subsequent run. The remaining acetal is distilled in vacuum without the column. It is sometimes necessary to remove traces of sodium acetate prior to distillation by renewed precipitation with ether.

Di-n-butoxy-dihydrofuran. B.p.15 $127-128^{\circ}$, yield $16.0 \mathrm{~g}=75 \%$.

\begin{tabular}{|c|c|c|c|}
\hline$U_{3}$ & $\begin{array}{r}\text { Calc } \\
\text { Found }\end{array}$ & $\begin{array}{l}\text { C } 67.26 \\
\text { " } 66.59\end{array}$ & $\begin{array}{ll}\text { H } \quad 10.35 \\
》 \quad 10.17\end{array}$ \\
\hline
\end{tabular}

$188 \mathrm{mg}$ of dibutoxy-dihydrofuran were boiled with $2 \mathrm{ml}$ of $0.01 \mathrm{~N}$ sulfuric acid and $2 \mathrm{ml}$ of alcohol for 2 minutes, and the bis-phenylhydrazone of malealdehyde prepared in the usual manner. Yield of crude product $164 \mathrm{mg}=71 \%$. Yield after recrystallization $94 \mathrm{mg}=41 \%, \mathrm{~m} \cdot \mathrm{p} \cdot 170-172^{\circ}$.

$$
\mathrm{C}_{16} \mathrm{H}_{16} \mathrm{~N}_{4} \quad \text { Calc. } \mathrm{N} 21.21 \quad \text { Found } \mathrm{N} 21.34
$$

Diisoamyloxy-dihydrofuran. B. p.0.6 $_{\cdot 0} 101-103^{\circ}$, yield $15.5 \mathrm{~g}=64 \%$.

$$
\begin{array}{lllllll}
\mathrm{C}_{14} \mathrm{H}_{26} \mathrm{O}_{3} & \text { Calc. } & \mathrm{C} & 69.39 & \mathrm{H} & 10.82 & \text { Mol. wt. } 242.3 \\
& \text { Found } & 69.53 & \text { " } & 10.54 & \text { " } & 230
\end{array}
$$

$169 \mathrm{mg}$ of diisoamyloxy-dihydrofuran were boiled with $1 \mathrm{ml}$ of $\mathrm{N}$ sulfuric acid and 1 $\mathrm{ml}$ of alcohol for one minute and the bis-phenylhydrazone of malealdehyde prepared in the usual manner. Yield of crude product $114 \mathrm{mg}=62 \%$. Yield after recrystallization $56 \mathrm{mg}=31 \%$, m. p. $169-170^{\circ}$.

$$
\mathrm{C}_{16} \mathrm{H}_{16} \mathrm{~N}_{4} \text { (264) Calc. N } 21.21 \quad \text { Found } \mathrm{N} 21.32
$$


Diisopropoxy-dihydrofuran. In the preparation of this acetal, it was necessary to add the bromine in a solution of carbon tetrachloride because of the ease with which isopropyl alcohol is oxidized by bromine. Furthermore, the acetic acid was removed by shaking the reaction mixture with $300 \mathrm{ml}$ of ether and two portions of potassium carbonate (each $50 \mathrm{~g}$ ) for 10 and 30 minutes, respectively. B. $p_{15} 85-90^{\circ}$, yield $13.0 \mathrm{~g}=70 \%$.

$$
\begin{array}{llllll}
\mathrm{C}_{10} \mathrm{H}_{18} \mathrm{O}_{4} & \text { Calc. } & \text { C } 64.51 & \mathrm{H} 9.74 & \text { Mol. wt. } 186.2 \\
& \text { Found } & 64.98 & * 9.73 &
\end{array}
$$

Diisopropoxy-dihydrofuran turns yellow after some days. This may, however, be due to a small amount of an impurity.

$209 \mathrm{mg}$ of the acetal were hydrolyzed as described for the butoxy derivative. Yield of crude malealdehyde-bisphenylhydrazone $150 \mathrm{mg}=51 \%$. Yield after recrystallization $117 \mathrm{mg}=40 \%$, m. p. $169-170^{\circ}$.

$$
\mathrm{C}_{16} \mathrm{H}_{16} \mathrm{~N}_{4} \text { (264) } \quad \text { Calc. } \mathrm{N} 21.21 \quad \text { Found } \mathrm{N} 21.25
$$

Molar refractions of dialkoxydihydrofurans

$2 \mathrm{ml}$ of the dialkoxydihydrofuran were redistilled carefully at $12 \mathrm{~mm}$. The middle fraction (about $1 \mathrm{ml}$ ) was collected and employed for the determination of the density, and the refractive index. The results are tabulated below.

\begin{tabular}{|l|c|c|c|c|c|c|}
\hline Dihydrofuran & B.p.12 & $d_{4}^{20}$ & $n_{\mathrm{D}}^{20}$ & $M_{\mathrm{D}}$ & $M_{\mathrm{D}}$ calc. & $\Delta$ \\
\hline & & & & & & \\
Dimethoxy- & $51^{\circ}$ & 1.0713 & 1.4328 & 31.56 & 32.17 & -0.61 \\
Diethoxy- & $\mathbf{7 1 - 7 3 ^ { \circ }}$ & 1.0019 & 1.4309 & 40.86 & 41.41 & -0.55 \\
Diisopropoxy- & $83-84^{\circ}$ & $\mathbf{0 . 9 5 3 9}$ & 1.4284 & 50.27 & 50.64 & -0.37 \\
Di- $n$-butoxy- & $118-120^{\circ}$ & 0.9436 & 1.4371 & 59.51 & 59.88 & -0.37 \\
Diisoamyloxy- & $130-131^{\circ}$ & 0.9267 & 1.4380 & 68.67 & 69.11 & -0.44 \\
\hline
\end{tabular}

\section{SUMMARY}

The general applicability of the alkoxylation method in the furan series has been investigated. 2,5-Diisopropoxy-, 2,5-di- $n$-butoxy- and 2,5-diisoamyloxy-2,5-dihydrofuran were prepared. 2-methoxy-5-acetoxy-2,5-dihydrofuran was isolated as a by-product from the methoxylation of furan. Other methods for the preparation of dihydrofurans by oxidation of furans have been discussed.

We are indebted to Mr. O. Rosenlund Hansen and Miss Muriel Schmidt for the microanalyses.

During the investigation we have received financial aid from Det teknisk-videnskabelige Forskningsraad (N. Clauson-Kaas and J. Fakstorp) and from Kemisk Vork Køge A/S, Copenhagen (F. Limborg). 


\section{REFERENCES}

1. Clauson-Kaas, N. Kgl. Danske Videnskab. Selskab, Mat.fys. Medd. 24 (1947) (6).

2. Clauson-Kaas, N., and Limborg, F. Acta Chem. Scand. 1 (1947) 619.

3. Meinel, K. Ann. 516 (1935) 231.

4. Clauson-Kaas, N., and Fakstorp, J. Acta Chem. Scand. 1 (1947) 415.

5. Clauson-Kaas, N. Acta Chem. Scand. 1 (1947) 379.

6. Buu-Hoï, Ng., and Lecocq, J. Compt. rend. 222 (1946) 1441.

7. Marquis, R. Ann. chim. (8) 4 (1905) 196.

8. Vargha, L., Ramonczai, J. and Bite, P. Nature 159 (1947) 744.

9. Clauson-Kaas, N., and Fakstorp, J. Acta Chem. Scand. 1 (1947) 210.

Received January 9, 1948. 